\title{
Corporate carbon performance data: Quo vadis?
}

\section{Timo Busch (iD) | Matthew Johnson (iD) | Thomas Pioch}

School of Business, Economics, and Social Science, University of Hamburg, Hamburg, Germany

\section{Correspondence}

Timo Busch, Business, Economics, and Social Science, University of Hamburg, Von-Melle-Park 9, Hamburg 20146, Germany.

Email: timo.busch@uni-hamburg.de

Editor Managing review: Charles Corbett

\begin{abstract}
Corporate carbon performance (CCP) has become a central topic in political, financial, and academic domains. At the same time, several characteristics of CCP data, including comparability and consistency, remain unresolved. The literature has extensively covered issues regarding the comparability of CCP data from a firm-internal perspective. However, it has not yet examined the consistency of CCP data between third-party data providers. This article investigates the degree of $\mathrm{CCP}$ data consistency between third-party providers according to three dimensions: scope (i.e., direct and indirect emissions), scheme (i.e., mandatory and voluntary reporting schemes), and source (i.e., data stemming from corporate reports and from third-party estimation methods). The results reveal that data on direct emissions are more consistent than data on indirect emissions, and they are especially inconsistent for Scope 3. Second, mandatory and voluntary reporting schemes do not substantially improve the consistency of CCP data, which is surprising. Third, third-party estimations are less consistent as compared to data stemming directly from corporate reports; however, the combination of Scopes 1 and 2 third-party estimated data raises consistency levels. On the basis of these results, we conclude the following key implications: academic researchers must be mindful of the consistency of CCP data, because it can significantly affect empirical results, corporate management should avoid situations where different CCP data are communicated externally, investors should engage firms to follow a standardized approach, data providers should increase the transparency about their estimation methods, and policy makers need to be aware of the importance of a sound and standardized methodology to determine CCP.
\end{abstract}

\section{KEYWORDS}

climate change, corporate carbon performance, data consistency, estimation method, industrial ecology, third-party provider

\section{1 | INTRODUCTION}

Corporate carbon performance (CCP) has emerged as a focal point in many domains: accounting and reporting schemes have been developed; a variety of actors in political, academic, and business spheres use these data; and business-related carbon emissions are considered increasingly relevant for investors' market valuation of firms (Griffin, Lont, \& Sun, 2017; Hahn, Reimsbach, \& Schiemann, 2015; Matsumura, Prakash, \& VeraMunoz, 2014). This momentum building can be observed in the following example: the European Union (EU) released an action plan on "Financing Sustainable Growth" (EU Commission, 2018a), which highlights the role of financial markets for climate change mitigation. Recently, a Task Force on Climate-related Financial Disclosures (TCFD, 2017) published its recommendations on disclosing information about the financial risks and opportunities presented by climate change.

To set realistic carbon reduction targets at the firm-level and measure progress, corporate managers, financial investors, academic researchers, and policymakers require comprehensive and high-quality CCP data. However, current CCP data reveal severe shortcomings in both regards. In

This is an open access article under the terms of the Creative Commons Attribution License, which permits use, distribution and reproduction in any medium, provided the original work is properly cited.

(c) 2020 The Authors. Journal of Industrial Ecology published by Wiley Periodicals LLC on behalf of Yale University 
terms of comprehensiveness, CCP data are typically available for large listed companies only, neglecting significant economic areas, such as supply chains and small and medium-sized enterprises. In terms of quality, the literature has extensively covered how companies should gather and report CCP data from a firm-internal perspective (Downie \& Stubbs, 2013; Hahn et al., 2015), but has assessed only to a limited extent the consistency of CCP data across firms from an external stakeholder perspective (Andrew \& Cortese, 2011; Marland, Buchholz, \& Kowalczyk, 2013; Matisoff, Noonan, \& O'Brien, 2013; Stanny, 2018).

Furthermore, the consistency of CCP data between third-party data providers has not yet been analyzed in a systematic manner. Third-party data providers, including Bloomberg, CDP, ISS Ethix, MSCI, Sustainalytics, Thomson Reuters, and Trucost, have established extensive datasets covering thousands of firms' carbon emissions over many years. We match this provided data over multiple years and address the following research questions: (RQ1) How consistent are CCP data between different third-party data providers in general?; (RQ2) To what extent do mandatory and voluntary reporting schemes affect the consistency of CCP data between third-party providers?; and (RQ3) How consistent are estimation methods between third-party providers?

We applied correlation analyses to measure consistency of CCP data offered by major third-party data providers. The analyses distinguish three dimensions of CCP data: scope (i.e., direct and indirect emissions), scheme (i.e., mandatory and voluntary reporting schemes), and source (i.e., data stemming from corporate reports and from third-party estimations). The results show that the consistency of CCP data is high for direct emissions, and progressively decreasing in Scope 2 to 3 indirect emissions. Subsequently, the results highlight that mandatory and voluntary reporting schemes do not substantially improve the consistency of CCP data between data providers. This is especially surprising for mandatory reporting schemes, that is, the EPA's Greenhouse Gas Reporting Program (GHGRP) and EU Emission Trading Scheme (ETS), as they prescribe the strict adherence to specific accounting and reporting methods. Third, data stemming from corporate reports is more consistent than data generated from third-party estimations; however, consistency of third-party estimations increases significantly when Scopes 1 and 2 are combined. From these results, we derive several implications for academic research, corporate management, financial markets, data providers, and policymaking.

\section{2 | LITERATURE REVIEW ON CCP DATA}

Previous literature has covered firm-internal drivers, motivations, and efforts to account and disclose CCP data. The main drivers include regulatory pressures, firm reputation, greater transparency, risk mitigation, and improving financial performance (Busch \& Hoffmann, 2011; Busch \& Lewandowski, 2018; Damert, Paul, \& Baumgartner, 2017; Hahn et al., 2015; Kolk, Levy, \& Pinkse, 2008; Luo, Lan, \& Tang, 2012; Reid \& Toffel, 2009). The disclosure of CCP data "potentially reduces the principal-agent problem of asymmetric information by increasing transparency" (Hahn et al., 2015 , p. 86). As such, companies disclose CCP data through voluntary channels-for example, sustainability reports or CDP-as a way to signal investors and other stakeholders of their superior firm performance (Clarkson, Li, Richardson, \& Vasvari, 2008; Diaz-Rainey, Robertson, \& Wilson, 2017). However, several issues remain pertaining to the quality of CCP data.

\subsection{The quality of CCP data}

In an attempt to increase the quality of CCP data, the GHG Protocol was released over 15 years ago as a standardized framework in accounting and reporting GHG emissions in organizations, including entire operations and value chains (WRI/WBCSD, 2004). Since 2008, the CDP encourages all companies to use the GHG Protocol to increase comparability (CDP, 2017). However, the comprehensiveness and quality of CCP data remain a major issue to this day (Matisoff et al., 2013; Stanny, 2018).

Hummel and Schlick (2016) describe several desirable characteristics of data quality. These include verifiability, reliability, comparability, and consistency. In terms of data verifiability and reliability (i.e., the data are accurate, fair, and true), the literature has extensively covered how firms internally gather and report CCP data, for example, examining the drivers and motives to report according to guidelines, such as the Greenhouse Gas Protocol (Downie \& Stubbs, 2013; Hahn et al., 2015; Schaltegger \& Csutora, 2012). Hummel and Schlick (2016) discover an interesting finding when it comes to data reliability. The authors find that superior sustainability performers choose to disclose high-quality, timely sustainability reports, whereas poor sustainability performers disguise their true performance by releasing low-quality, less frequent reports (Hummel \& Schlick, 2016). The latter finding was confirmed in a recent study showing how companies conceal information and employ various impression management strategies, including making excuses and delays, to hide poor performance (Talbot \& Boiral, 2018).

The literature acknowledges the differences and limitations of CCP data comparability across firms. Andrew and Cortese (2011) examined different accounting methodologies from various reporting schemes, and concluded that CCP data will remain incomparable and ultimately unreliable for an unforeseeable future. Matisoff et al. (2013) studied the degree of data convergence in CDP from 2003 to 2010, examining trends in disclosure, transparency, and comparability of corporate carbon emission data over this period. They concluded as the amount of companies reporting increases, the comparability between disclosures increases in Scopes 1 and 2, but not in Scope 3. However, as more companies disclose CCP data, Matisoff et al. (2013) observed a decrease in the percentage of reports becoming available, hence less reliable for public scrutiny. 
However, the consistency of CCP data between third-party providers has not been empirically investigated. Third-party data providers generate large datasets based on data from corporate reports and their own estimation methods. The latter approach is used primarily when companyreported data are not available. Thus, our first research question deals with the general level of consistency for CCP data between different thirdparty data providers.

\section{2 | Mandatory and voluntary reporting schemes}

Most companies report CCP data according to either mandatory or voluntary reporting schemes (Andrew \& Cortese, 2011). Several regions and nations require energy-intensive companies to report annual carbon emissions of specific facilities according to a mandatory reporting scheme. For example, the European Union Emission Trading System (EU ETS) covers approximately 11,000 facilities, including large power stations and manufacturing plants, which cover around 45\% of the GHG emissions in the European Union (EU Commission, 2018b). The U.S. Environmental Protection Agency (USEPA, 2018) requires facilities that emit more than 25,000 metric tons of $\mathrm{CO}_{2}$-equivalent $\left(\mathrm{CO}_{2} \mathrm{e}\right)$ emissions per year to disclose their annual emissions through the Greenhouse Gas Reporting Program (GHGRP). This program covers over 8,000 facilities and roughly 50\% of the total U.S. emissions (USEPA, 2017).

Mandatory reporting schemes were established to monitor facility-level CCP data according to strict, formal rules for accounting and reporting (Perrault \& Clark, 2010; Sullivan \& Gouldson, 2012). Since these schemes consider carbon emissions at facility level, all covered emissions related to Scope 1. It can be assumed that emission data reported under a mandatory regulation is regarded as highly standardized and consistent. Nevertheless, it has been found that other aspects, such as reporting boundaries and emission factors, can be manipulated and, thus, may affect data comparability (Dragomir, 2012; Sullivan \& Gouldson, 2012; Talbot \& Boiral, 2018).

Beyond mandatory schemes, several voluntary carbon reporting initiatives have emerged. Voluntary reporting schemes have an advantage over mandatory reporting, as "voluntary disclosure programs like the CDP may allow firms to engage with stakeholders such as investors and employees more directly than mandatory reporting requirements and serve as a way to improve internal management of GHGs" (Matisoff et al., 2013, 297). Undoubtedly, the most successful voluntary initiative is the CDP (formerly the Carbon Disclosure Project) with more than 2,400 companies disclosing CCP data (CDP, 2017). Since 2003, the CDP collects data via an annual questionnaire from major corporations in various capital markets. Despite its success, several studies have raised concerns regarding the quality of data gathered and published by the CDP (CDP, 2010; Andrew \& Cortese, 2011; Matisoff et al., 2013; Stanny, 2018).

The distinction between mandatory and voluntary reporting schemes is relevant in this article because they differ in scope and scheme. On the one hand, mandatory reporting schemes are stricter in terms of accounting methodology, but they are limited to direct emissions (Scope 1) and pertain to individual facilities (i.e., not entire firms). On the other hand, voluntary disclosures encourage companies to report both direct (Scope 1) as well as indirect emissions (Scopes 2 and 3). While such schemes encourage firms to report emissions for the entire firm and its value chain, they are much more lenient in accounting methodology, and this makes the comparability between firms difficult (Perrault \& Clark, 2010). We explore the extent that mandatory and voluntary reporting schemes affect the consistency of CCP data between third-party providers.

\section{3 | Third-party estimation methods}

Although the number of firms disclosing their carbon emissions has increased over the years, many companies still do not report their carbon emissions. In those cases, third-party providers can use their own estimation methods to fill these data gaps. Such estimation methods are also essential in cases where investors and other stakeholders would like to assess the carbon footprint of supply chains since CCP data are typically scarce further along the supply chain.

The literature suggested three general approaches for estimation methods (Goldhammer, Busse, \& Busch, 2017). First, a process analysis (PA) approach using primary and secondary production process data and the associated carbon emissions (Minx et al., 2009; Suh \& Huppes, 2009; Wiedmann \& Minx, 2008). This approach has been used extensively to estimate carbon footprints on a product level (Burkhardt, Heath, \& Cohen, 2012; Dolan \& Heath, 2012; Mazor, Mutton, Am Russell, \& Keoleian, 2011) as well as on a firm level (Block et al., 2011; Gooding, 2012; Lee \& Cheong, 2011). This approach is very detailed and requires a lot of specific information. At the same time, boundaries for proper calculation need to be established, which may result in a lack of completeness of the estimation (Minx et al., 2009; Suh \& Huppes, 2009).

Second, input-output analysis (IOA) uses the input-output tables of national accounts to allocate the carbon emissions of the economy to smaller units on a sectoral basis. This approach has been applied to carbon footprint calculation at the firm level (Minx et al., 2009), for example, by ascribing emissions to individual firms according to their relative proportion of the firm's sales compared the sectors overall sales numbers. Assessing emissions per unit of turnover appears to be an appropriate frame of reference since it allows the most comparable analysis of individual CCP in the absence of any further company specific information. This method can be extended to a multi-regional input-output (MRIO) analysis (e.g., Lundie, Wiedmann, Welzel, \& Busch, 2019). Therefore, MRIO analysis can also capture the Scope 3 emissions along the entire supply chain (Huang, Lenzen, Weber, Murray, \& Matthews, 2009). The estimations resulting from MRIO analyses represent averages and do not consider firm specific aspects (Minx et al., 2009). 
Third, hybrid approaches merge the strengths of both PA and IOA. They have been coined the state of the art for carbon footprinting (Wiedmann, 2009). The Norwegian "Klimakost" model is one example of a hybrid approach being applied to companies, municipalities and other organizations (Larsen, Solli, \& Pettersena, 2012).

Third-party data providers combine and extend these approaches to estimate CCP data. ISS-Ethix estimates CCP data with the help of more than 800 sub-sector specific models, where every sub-sector is analyzed to identify emission predictors (ISS-Ethix Climate Solutions, 2019). MSCI ESG employs different estimation methods to determine CCP, for example, based on a company's previous emission intensities (MSCI ESG Research, 2019). Sustainalytics uses more than 80 different estimation models to determine Scope 1 and 2 emissions, which are based on historical company data and non-linear regressions (Sustainalytics, 2019). The 80 different models are then calculated as average emissions per million USD revenue. Thomson Reuters ESG applies one of three different estimation methods to determine Scope 1 and 2 emissions in the following order, according to data availability: (a) previously reported emissions, (b) energy consumption, and (c) median emissions of industry or business sector (Thomson Reuters ESG, 2019). Finally, Trucost uses its own Extended Environmental Input-Output (EEIO) model based on industry-specific environmental impact data, and the flow of goods and services between economic sectors (Trucost ESG Analysis, 2019).

While the individual approaches differ, the third-party providers do share some commonalities in the estimation methods. Thomson Reuters ESG and Sustainalytics, for example, use similar business metrics to calculate industry average CCP data in the form of intensities and then convert those back to absolute emissions. ISS-Ethix and Trucost base their estimations on sector and country-specific models utilizing industry input-output tables. It appears that most third-party providers use industry-based methods over location-based methods. While various estimation methods can still yield different results, a high degree of consistency between third-party data estimations would be desirable. Thus, our third research question examines how consistent estimation methods are between third-party providers.

\section{3 | METHOD}

\section{1 | Sample selection}

In order to establish a representative sample, we selected third-party data providers based on two criteria. The first criterion is data type-data providers must offer company-wide $\mathrm{CO}_{2}$ e emission data in Scopes 1 and 2 as a minimum requirement, and if available, Scope 3 emissions. We excluded any data providers that only offered carbon scores or climate ratings to represent CCP information. The second criterion is data rangethird-party providers must offer historical data for more than 5 years of same-firm data. Additionally, they should provide a global coverage of firms. We excluded any third-party data providers with only a few years of coverage, including those that have discontinued this service, as well as third-party providers focusing on a single country.

We derived a list of major third-party CCP data providers, including Bloomberg, CDP, ISS Ethix, MSCI, Sustainalytics, Thomson Reuters ESG, and Trucost. Table 1 shows the data type, timespan, coverage of companies having reported data, total company coverage, and additional details of each provider. We obtained this information through the providers' websites and downloadable factsheets. When information was not available, we contacted the data providers directly, and asked them to provide supplemental information.

We matched CCP data from different providers for the same firm using the International Securities Identification Number (ISIN), resulting in a sample of 15,485 firms between 2005 and 2016. From this sample, we were able to draw firm-year observations where at least two providers can be compared: 50,793 observations (Scope 1), 50,609 observations (Scope 2), and 12,355 observations (Scope 3). The data correspond to the years of actual emissions. We calculated the pairwise Pearson correlation coefficient to analyze the strength of the relationship and thus the degree of consistency between two individual data providers. In addition, we also calculated the Spearman rank correlation coefficients, as they are less affected by potential outliers in the data.

In order to scrutinize the effects of mandatory emission reporting schemes, we utilized facility level carbon emissions as provided by the USEPA GHGRP and the EU ETS. We matched these facility emissions to their respective primary owners on a firm level. This allowed us to calculate aggregated facility emissions that are matched to one company. The USEPA GHGRP database provides information on the ownership structure of each facility through company names and the ownership percentage. Using these company names, we matched facilities to a single company ISIN. In cases where a facility has multiple owners, we considered the primary owner as the principal owner for our analysis. For the EU ETS, the World Carbon Market Database (WCMD, 2019) offers facility to company matching tables, including the ISINs. With the ISIN as the identifier, we were able to compare aggregated facility level emissions with CCP data from third party providers.

\section{2 | Descriptive statistics and data handling}

The descriptive statistics illustrate a heterogeneous picture for all emission scopes (see Table 2). Maximum values, means, and standard deviations vary substantially across providers for all scopes, pointing toward high levels of inconsistency. The large differences for maximum values made it 
TABLE 1 Overview of data providers

\begin{tabular}{|c|c|c|c|c|c|}
\hline Data provider & Data type & Timespan ${ }^{*}$ & $\begin{array}{l}\text { Coverage of } \\
\text { reported data }\end{array}$ & Total coverage & Additional details \\
\hline Bloomberg & $\mathrm{CO}_{2} \mathrm{e}$ Scopes 1-3 & 2005-present & N.A. & $\begin{array}{l}\sim 10,000 \\
\quad \text { companies }\end{array}$ & $\begin{array}{l}\text { Combines data from } \\
\text { several sources. }\end{array}$ \\
\hline ISS Ethix & $\mathrm{CO}_{2}$ e Scopes 1-3 & 2005-present & $\begin{array}{l}\sim 4,000 \\
\quad \text { companies }\end{array}$ & $\begin{array}{l}\sim 25,000 \\
\quad \text { companies }\end{array}$ & $\begin{array}{l}\text { Includes company } \\
\text { reported data, } \\
\text { third-party data } \\
\text { and estimations. } \\
\text { Additional } \\
\text { forward-looking } \\
\text { climate risk data } \\
\text { provided. }\end{array}$ \\
\hline Sustainalytics & $\mathrm{CO}_{2}$ e Scopes 1-2 & 2010-present & $\begin{array}{l}\sim 2,000 \\
\quad \text { companies }\end{array}$ & $\begin{array}{l}\sim 11,000 \\
\quad \text { companies }\end{array}$ & $\begin{array}{l}\text { Includes company } \\
\text { reported data, } \\
\text { third-party data } \\
\text { and own } \\
\text { estimations. } \\
\text { Carbon Solutions } \\
\text { Suite }{ }^{\circledR} \text { provides } \\
\text { additional } \\
\text { information. }\end{array}$ \\
\hline Trucost & $\mathrm{CO}_{2} \mathrm{e}$ Scopes 1-3 & 2005-present & $\begin{array}{l}\sim 1,800 \\
\quad \text { companies }\end{array}$ & $\begin{array}{l}\sim 14,000 \\
\quad \text { companies }\end{array}$ & $\begin{array}{l}\text { Includes company } \\
\text { reported data, } \\
\text { third-party data } \\
\text { and own } \\
\text { estimations. } \\
\text { Estimation of } \\
\text { Scope } 3 \text { emissions. }\end{array}$ \\
\hline
\end{tabular}

Note. Table 1 contains an overview of major $\mathrm{CO}_{2}$ e emission data providers. The information in this table is derived from the respective database documentation of each provider. The timespan corresponds to the years of emissions and not years of reports.

necessary to further investigate potential outliers. Since Pearson correlation coefficients are sensitive to extreme outliers, we manually investigated some of the extreme outliers, for example, 88,000,000,000 t $\mathrm{CO}_{2}$ e emissions indicated as the annual Scope 3 emissions of one company, which is more than the annual anthropogenic emissions. The additional analysis of the descriptive statistics in Table 2 led to the conclusion that the dataset had to be adjusted by removing severe outliers. To minimize the effect of potential errors in the data, we removed outliers by deleting $0.05 \%$ of the observations at the low and high end of the distribution for each provider. This adjusted dataset shows significantly higher correlations in all scopes and was used as a basis for further analyses.

Several data providers were not included in specific analyses due data availability reasons. ISS Ethix could not be included in many analyses, as they only offered combined Scope 1 and 2 data and estimated Scope 3 data. Sustainalytics was left out of Scope 3 analyses, as they do not provide Scope 3 data at all. Bloomberg does not reveal if the data stem from company reports or third-party estimates; therefore Bloomberg only included in general analyses (Bloomberg Finance L.P., 2019). The analyses for third-party estimated Scope 3 data was limited to two providers offering related data, ISS Ethix and Trucost. 
TAB LE 2 Descriptive statistics of Scopes 1, 2 and $3 \mathrm{t} \mathrm{CO}_{2}$ e emissions

\begin{tabular}{|c|c|c|c|c|c|}
\hline Provider (Scope 1) & Observations & Min & Max & Mean & Std. Dev. \\
\hline Bloomberg & 11,688 & .1 & $203,160,000$ & $3,436,052$ & $13,234,181$ \\
\hline CDP & 12,368 & .1 & $461,661,074$ & $3,745,308$ & $15,776,920$ \\
\hline Sustainalytics & 31,244 & .2 & $571,928,834$ & $1,947,869$ & $12,496,509$ \\
\hline Thomson Reuters & 13,830 & .014 & $603,242,000$ & $4,057,620$ & $15,628,072$ \\
\hline Bloomberg & 11,459 & .1 & $30,900,000$ & 689,211 & $2,060,708$ \\
\hline CDP & 12,077 & 1.82 & $172,642,421$ & 701,218 & $3,058,222$ \\
\hline $\mathrm{MSCl}$ & 72,266 & 1 & $2,464,659,522$ & 260,294 & $9,223,938$ \\
\hline Sustainalytics & 31,327 & 1.82 & $29,184,916$ & 298,464 & $1,117,465$ \\
\hline Bloomberg & 7,060 & 1 & $917,117,000$ & $10,293,090$ & $58,544,006$ \\
\hline CDP & 6,868 & .61 & $88,059,800,000$ & $36,889,891$ & $1,141,664,189$ \\
\hline ISS Ethix & 55,408 & 0 & $2,025,229,344$ & $1,567,979$ & $21,395,923$ \\
\hline $\mathrm{MSCl}$ & 8,860 & 1 & $5,967,000,000$ & $14,057,086$ & $112,160,780$ \\
\hline Thomson Reuters & 8,421 & .2 & $1,112,720,049$ & $11,867,324$ & $62,246,568$ \\
\hline Trucost & 61,100 & .41 & $663,034,435$ & $1,459,358$ & $6,375,612$ \\
\hline
\end{tabular}

Note. Table 2 shows the descriptive statistics of the data as it is available directly from each provider. No alterations to the data were made. All emissions are measured in metric tons.

\section{4 | RESULTS}

With respect to RQ1, the results show that Scope 1 data are highly consistent between most data providers with an average Pearson correlation coefficient of 0.97 and average Spearman correlation coefficient of 0.95. As shown in Table 3, a general pattern emerges: Scope 1 data are more consistent than Scope 2 data, and Scope 2 data are more consistent than Scope 3 data, where the biggest inconsistencies can be found. The Spearman rank correlations exhibit similar patterns for all our analyses and generally confirm that low Pearson correlation levels are not exclusively the result of individual outliers in the data.

Additionally, we investigated the development of consistency between third-party providers over time (compare Figure S1 in the Supporting Information). The general expectation would be that third-party data providers gain experience in gathering and processing CCP data over time. However, the overall data consistency fails to improve substantially. On the contrary, data on Scope 3 emissions became even less consistent over time.

In answering RQ2, we use facility-level emission data from the EPA GHGRP and EU ETS. Table 4 shows the percentage these aggregated facility emissions constitute of the total Scope 1 emissions of the matched company. The sample includes only U.S. and EU facilities, since GHGRP is under U.S. regulation and EU ETS is under European directives. Parent companies of these facilities can, however, originate from other countries and have multiple facilities not covered by these schemes. Aggregated facility-level emissions reported by companies through the USEPA GHGRP constitute on average between 33.01\% and 41.74\% of a company's total reported Scope 1 emissions. For companies reporting emissions under the EU ETS, aggregated facility-level emissions constitute between $39.38 \%$ and $49.36 \%$ of companies' total reported Scope 1 emissions. This analysis shows that mandatory reported data based on facility level provide little help in generating greater consistency of CCP data, since the mandatory reported data cover only a portion of a company's overall Scope 1 emission profile. For example, Enbridge, Inc. operates one of the world's largest crude oil pipeline networks in Canada and the United States. The $\mathrm{CO}_{2}$ e emissions stated in the sustainability report differs from what the company reports to the USEPA GHGRP for two reasons. First, the sustainability report includes global Scope 1 emissions, while the USEPA GHGRP report only covers U.S. facilities. Second, the calculation methods they used for the facilities covered by the EPA GHGRP is different than for the facilities outside of this regulation and facilities with less than $25,000 \mathrm{t} \mathrm{CO}_{2}$ e emissions annually (Enbridge Inc., 2017).

Given the results in Table 4, we expected a low consistency measured through correlation coefficients between aggregated facility level emissions and company Scope 1 emissions. The results in Table 5 confirm our expectations, and reveal that facility level emissions are not strongly correlated with companies' overall Scope 1 emissions. Facility emissions covered by mandatory reporting schemes are typically lower as the 
TABLE 3 Adjusted CCP data

\begin{tabular}{|c|c|c|c|c|c|c|}
\hline Scope 1 & Bloomberg & CDP & $\mathrm{MSCl}$ & Sustainalytics & T. Reuters & Trucost \\
\hline \multirow[t]{2}{*}{ Bloomberg } & 1 & $0.9839^{*}$ & $0.9795^{*}$ & $0.9255^{*}$ & $0.9901^{*}$ & $0.9451^{*}$ \\
\hline & $(11,679)$ & $(5,482)$ & $(9,007)$ & $(5,964)$ & $(7,997)$ & $(9,405)$ \\
\hline CDP & $(5,482)$ & $(12,359)$ & $(9,200)$ & $(6,005)$ & $(7,456)$ & $(9,040)$ \\
\hline $\mathrm{MSCl}$ & $0.9912^{*}$ & $0.9621^{*}$ & 1 & $0.9013^{*}$ & $0.9759^{*}$ & $0.8899^{*}$ \\
\hline Sustainalytics & $(5,964)$ & $(6,005)$ & $(24,834)$ & $(31,216)$ & $(6,651)$ & $(20,967)$ \\
\hline \multirow[t]{2}{*}{ T. Reuters } & $0.9918^{*}$ & $0.9746^{*}$ & $0.9876^{*}$ & $0.9637^{*}$ & 1 & $0.9496^{*}$ \\
\hline & $(7,997)$ & $(7,456)$ & $(10,720)$ & $(6,651)$ & $(13,818)$ & $(11,623)$ \\
\hline Trucost & $0.9845^{*}$ & $0.9598^{*}$ & $0.9615^{*}$ & $0.8860^{*}$ & $0.9861^{*}$ & 1 \\
\hline Bloomberg & $(11,449)$ & $(5,303)$ & $(8,859)$ & $(5,905)$ & $(7,784)$ & $(9,235)$ \\
\hline \multirow[t]{2}{*}{ CDP } & $0.9647^{*}$ & 1 & $0.9768^{*}$ & $0.9869^{*}$ & $0.9805^{*}$ & $0.9518^{*}$ \\
\hline & $(5,303)$ & $(12,066)$ & $(8,977)$ & $(5,858)$ & $(7,241)$ & $(8,828)$ \\
\hline \multirow[t]{2}{*}{$\mathrm{MSCl}$} & $0.9393^{*}$ & $0.8715^{*}$ & 1 & $0.9049^{*}$ & $0.9626^{*}$ & $0.8423^{*}$ \\
\hline & $(8,859)$ & $(8,977)$ & $(72,240)$ & $(24,958)$ & $(10,459)$ & $(38,817)$ \\
\hline \multirow[t]{2}{*}{ Sustainalytics } & $0.9022^{*}$ & $0.9418^{*}$ & $0.8780^{*}$ & 1 & $0.9167^{*}$ & $0.8566^{*}$ \\
\hline & $(5,905)$ & $(5,858)$ & $(24,958)$ & $(31,297)$ & $(6,601)$ & $(21,075)$ \\
\hline \multirow[t]{2}{*}{ T. Reuters } & $0.9208^{*}$ & $0.9389^{*}$ & $0.8675^{*}$ & $0.7956^{*}$ & 1 & $0.9251^{*}$ \\
\hline & $(7,784)$ & $(7,241)$ & $(10,459)$ & $(6,601)$ & $(13,200)$ & $(11,179)$ \\
\hline \multirow[t]{2}{*}{ CDP } & $0.4137^{*}$ & 1 & $0.8499^{*}$ & $0.8049^{*}$ & $0.5835^{*}$ & \\
\hline & $(3,038)$ & $(6,862)$ & $(4,597)$ & $(4,089)$ & $(5,321)$ & \\
\hline \multirow[t]{2}{*}{$\mathrm{MSCl}$} & $0.8963^{*}$ & $0.4045^{*}$ & 1 & $0.8923^{*}$ & $0.6510^{*}$ & \\
\hline & $(4,215)$ & $(4,597)$ & $(8,856)$ & $(5,257)$ & $(7,517)$ & \\
\hline \multirow[t]{2}{*}{ T. Reuters } & $0.9864^{*}$ & $0.3956^{*}$ & $0.8907^{*}$ & 1 & $0.6320^{*}$ & \\
\hline & $(4,870)$ & $(4,089)$ & $(5,257)$ & $(8,414)$ & $(7,121)$ & \\
\hline \multirow[t]{2}{*}{ Trucost } & $0.5924^{*}$ & $0.2203^{*}$ & $0.3813^{*}$ & $0.5771^{*}$ & 1 & \\
\hline & $(5,731)$ & $(5,321)$ & $(7,517)$ & $(7,121)$ & $(61,040)$ & \\
\hline
\end{tabular}

Note. Table 3 shows the Pearson (lower left side) and Spearman (upper right side) correlation coefficients of our base sample which has been adjusted by removing the top and bottom $0.05 \%$ of observations.

*Indicates a significance level of $p<0.01$, number of observations in parentheses.

companies' overall Scope 1 emissions. For many companies, a majority of their Scope 1 emissions are not subject to the regulations. Thus, we conclude, mandatory reporting schemes do not help improve CCP data consistency. However, exceptions can be found for certain industries or companies with numerous high-emitting facilities that are covered by the mandatory schemes. For example, the energy company RWE has most of its power plants covered within the EU ETS. RWE had in 2016 facility level emissions reported under the EU ETS of 139 million metric tons, while third-party providers reported Scope 1 emissions of 154 million metric tons ( $90 \%$ of their global Scope 1 emissions).

Next to mandatory schemes we investigate the extent of how voluntary reporting schemes affect the consistency of CCP data between thirdparty providers. To measure this properly, we first established a baseline by investigating the correlations of CCP data indicated as companyreported (Table 6). We found that company-reported data increased the overall correlation levels in Scopes 1 and 2. Moreover, Table 6 reveals 
TA B LE 4 Aggregated facility emissions of a firm compared to their reported Scope 1 emissions

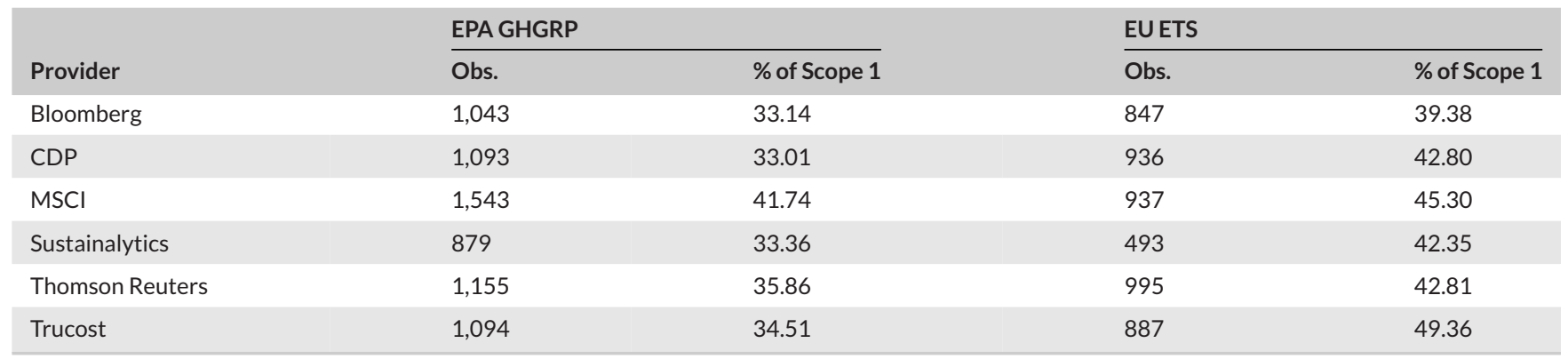

Note. Table 4 shows the facility level $\mathrm{CO}_{2}$ e emission data provided under the EPA GHGRP and the EU ETS, compared to the Scope 1 emission data from third party providers. For each provider, we show the share of emissions under the EPA GHGRP and the EU ETS of the total reported Scope 1 emissions.

TA B LE 5 Correlation between aggregated facility emissions and Scope 1 emissions from data providers

\begin{tabular}{|c|c|c|c|c|c|c|c|}
\hline & & Bloomberg & CDP & $\mathrm{MSCl}$ & Sustain. & T. Reuters & Trucost \\
\hline $\begin{array}{c}\text { Facility GHGs - } \\
\text { EPA GHGRP }\end{array}$ & $\begin{array}{l}\text { Pearson } \\
\text { correlation } \\
\text { coefficients }\end{array}$ & $0.5961^{*}(1,154)$ & $0.5728^{*}(1,214)$ & $0.6013^{*}(2,995)$ & $0.5636^{*}(2,166)$ & $0.5282^{*}(1,323)$ & $0.5940^{*}(2,517)$ \\
\hline $\begin{array}{l}\text { Facility GHGs - } \\
\text { EU ETS }\end{array}$ & $\begin{array}{l}\text { Spearman } \\
\text { rank } \\
\text { correlation } \\
\text { coefficients }\end{array}$ & $0.7727^{*}(942)$ & $0.8023^{*}(1,040)$ & $0.7589^{*}(1,380)$ & $0.7871^{*}(726)$ & $0.7635^{*}(1,252)$ & $0.7759^{*}(1,657)$ \\
\hline
\end{tabular}

Note. Table 5 shows the Pearson and Spearman correlation coefficients between the $\mathrm{CO}_{2}$ e emission data provided under the EPA GHGRP and the EU ETS and the third party providers in our study.

*Indicates a significance level of $p<0.01$, number of observations in parentheses.

that the combination of Scopes 1 and 2 into a single data item also yields higher correlation levels compared to Scope 2 alone. Consistency in Scope 3 emissions does not improve with company-reported data when compared to the overall findings (Table 3), which again reveals how difficult it is to properly gather these data.

Next, we created a subsample of company-reported data only for firms included in the CDP database. The findings show opposite trends when compared to the results in Table 6. We detected lower correlations for Scopes 1 and 2 . This suggests that reporting to the CDP can potentially cause further data inconsistency. One explanation for greater inconsistency may be related to different values presented as "company-reported data" (i.e., CDP and company reports), which providers have to choose. For example, in 2010, ConocoPhilips, the third-largest U.S. oil company, reported to the CDP emissions of 57,981,000 t $\mathrm{CO}_{2} \mathrm{e}$. Both TruCost and Bloomberg report this figure. At the same time, Thomson Reuters and $\mathrm{MSCl}$ must have relied on a different source of information in this case, since both report 26,618,645 $\mathrm{tCO}_{2} \mathrm{e}$ in their databases. Upon further investigation, we found that Thomson Reuters and $\mathrm{MSCl}$ based their data from the company revised value of 26.6 million metric tons of $\mathrm{CO}_{2}$ e published in a later report (ConocoPhilips, 2011).

Finally, RQ3 focusses on the consistency levels of estimation methods between third-party providers. Only three data providers offer third-party estimations on individual Scopes 1 and 2 emissions-MSCI, Sustainalytics, and Trucost. Thomson Reuters and ISS Ethix provide only combined Scope 1 and 2 figures for estimations. Moreover, ISS Ethix and Trucost are the only providers estimating Scope 3 emissions.

The results in Table 7 illustrate that-not surprisingly-correlation levels between data providers are lower when using only third-party estimations for both Scope 1 and 2 emissions. For example, the Pearson correlations of Scope 1 emissions between MSCl and Sustainalytics decrease from 0.99 (company-reported) to 0.77 (third-party estimates) and from 0.98 (company-reported) to 0.69 (third-party estimates) for Scope 2 emissions. When combining Scopes 1 and 2, the correlation levels improve for third-party estimated data even though the underlying estimation models used by the data providers differ considerably. The two estimations for Scope 3 emissions yield inconsistent results. In this case, $97 \%$ of observations differ by more than $10 \%$ between the two providers. These low correlations highlight the differences in third-party estimation results. An extreme example of this is the WH Group, the world's largest pork producer, where TruCost estimates 39,839,717 and ISS 6,707,330 t CO ${ }_{2}$ e Scope 3 emissions in 2015. 
TAB LE 6 CCP data from company reports

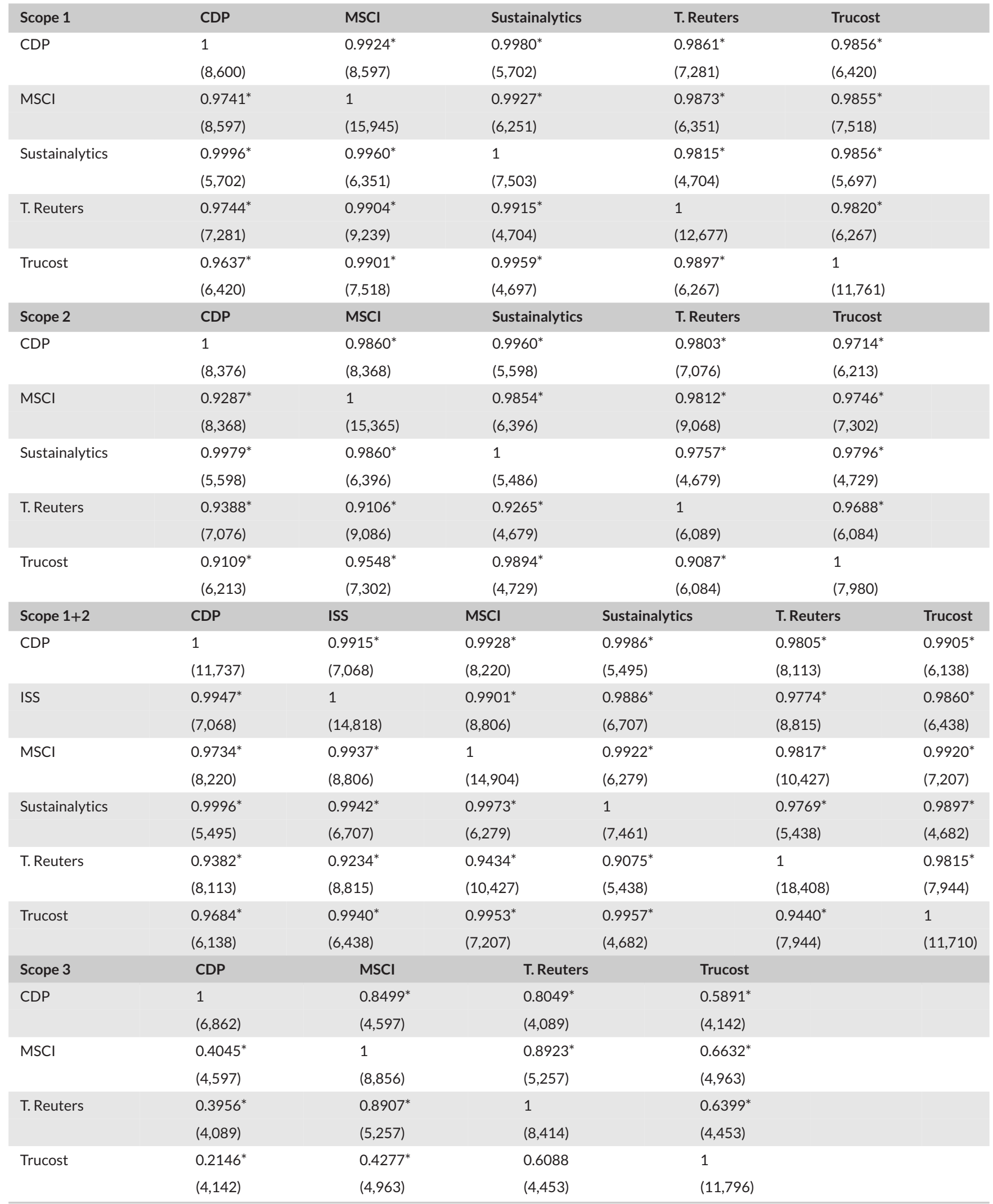

Note. Table 6 shows the Pearson (lower left side) and Spearman (upper right side) correlation coefficients between third party data providers. The data included in this table is limited to those observations that were clearly identified as originating from company reports by the provider.

"Indicates a significance level of $p<0.01$, number of observations in parentheses. 
TAB LE 7 CCP data from third-party estimations

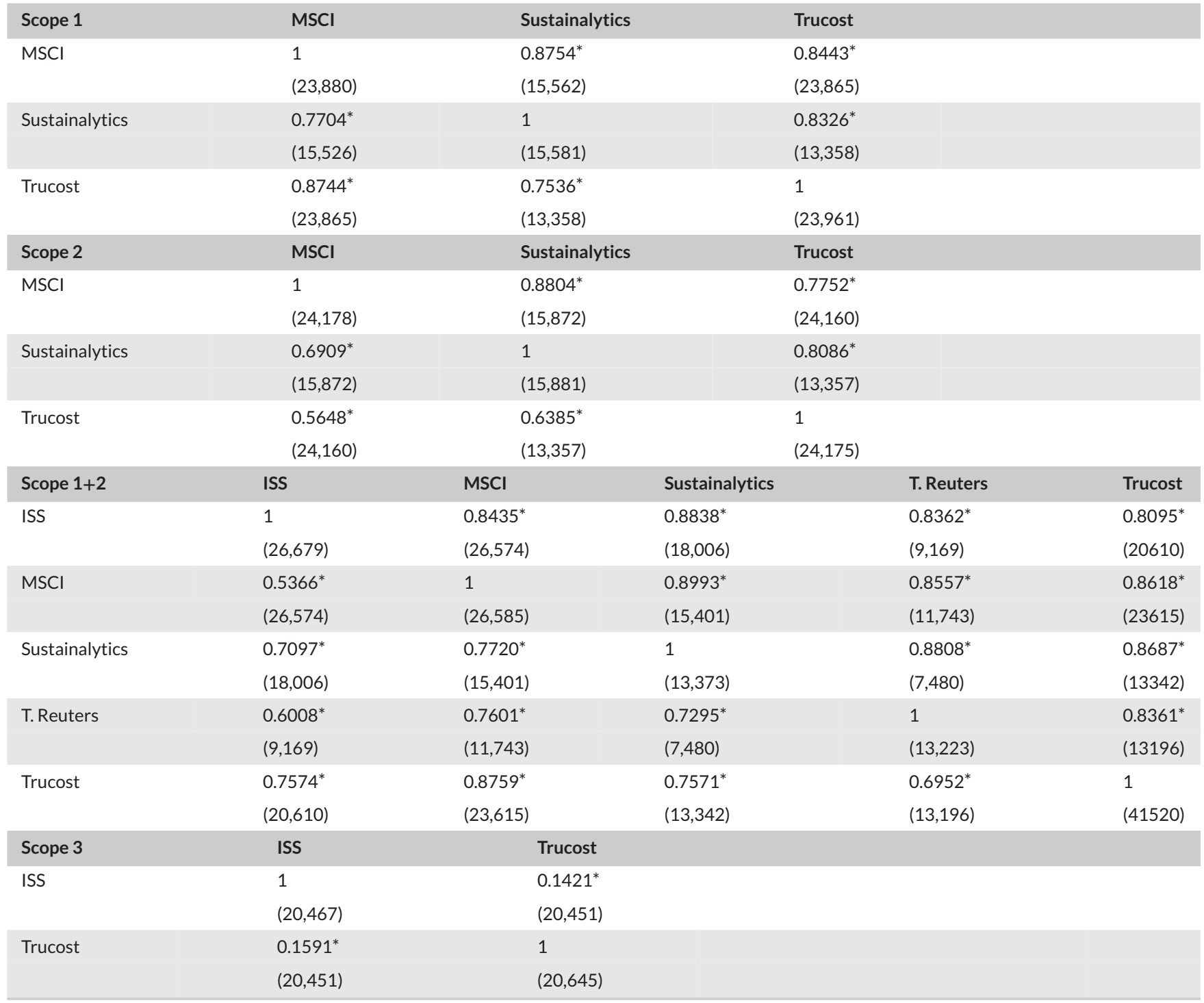

Note. Table 7 shows the Pearson (lower left side) and Spearman (upper right side) correlation coefficients between third party data providers. The data included in this table is limited to those observations that were clearly identified as originating from estimations by the provider.

*Indicates a significance level of $p<0.01$, number of observations in parentheses.

\section{5 | DISCUSSION}

The findings are important along three dimensions-scope, scheme, and source. According to scope, we observe as the complexity of carbon accounting increases from direct emissions (Scope 1) to indirect emissions (Scope 2 and 3), the consistency of CCP data between third-party providers decreases. The higher levels of data consistency in Scopes 1 and 2 is most likely a consequence of investors' requests for more transparent disclosure in these areas (Sullivan \& Gouldson, 2012). Thus, it seems essential to engage further in promoting and requesting firms to follow a standardized approach, even in the more complex Scope 3 realm (Matisoff et al., 2013).

According to reporting scheme, mandatory reporting schemes provide little benefit in generating higher consistency of CCP. Since these reporting schemes are based on facility-level data, they deliver an incomplete picture of companies' overall Scope 1 emissions. CCP data stemming from mandatory reporting schemes, including the USEPA GHGRP and EU ETS, cover between 33\% and 49\% of firms' Scope 1 carbon emissions. Additionally, CCP data stemming from voluntary reporting schemes do not improve data consistency. If firms report different values through different reporting channels, this creates further inconsistency. This is in line with previous research findings that the use of different data sources, that is, CDP and sustainability reports, leads to higher levels of inconsistency (Depoers, Jeanjean, \& Jérôme, 2016; Perrault \& Clark, 2010).

With respect to source, the consistency of third-party estimated data is lower when compared to data stemming from company reports. However, the combination of estimated data for Scope 1 and 2 emissions provides a surprising result of higher consistency. This outcome illustrates 
that the applied estimation methods can improve consistency to a certain extent. The biggest inconsistencies emerge for Scope 3 emissions-for both company reported as well as estimated data. More importantly, third-party providers do not provide transparent coverage of the categories of Scope 3 emissions. Thus, transparency of the Scope 3 sources will become important as more companies set reduction goals along supply chains according to science-based targets (Rekker, Humphrey, \& O’Brien, 2019).

These findings reveal two points for further discussion: (a) the value of fostering consistency and (b) the significance of third-party estimation methods. Fostering CCP data consistency is important for several reasons. First, consistent CCP data are a necessary requirement for a range of stakeholders in order to measure, analyze, and compare CCP data between firms and over time. Achieving high levels of CCP data consistency should ultimately create a "level playing field between firms and across industries" (Bowen \& Wittneben, 2011, p. 1029). Second, consistent CCP data are the foundation for thorough stress tests for banks and investors considering CCP data in investment decisions. For example, the TCFD (2017) has developed recommendations for climate-related financial disclosures for a better understanding of related financial risks. However, the TCFD (2017, p. 1) stresses the lack of consistent CCP data "hinders investors and others from considering climate-related issues in their asset valuation and allocation processes". Third, as more countries and regions implement carbon taxes and emission trading schemes, CCP data-notably beyond the already covered facility level data-will become more important from a financial perspective. Thus, fostering consistent CCP data covering all three emission scopes is essential for accurately assessing the business risks and opportunities in pathways toward decarbonization (Griffin et al., 2017; Stanny \& Ely, 2008).

Third-party estimations play an important role in the CCP data context, as data providers fill in data gaps when companies do not or only partially report emission data themselves. This is especially the case for companies that are not listed on stock exchanges, covering a majority of small and medium-sized enterprises (Bradford \& Fraser, 2008). As such, third-party estimations will continue to play a significant role. However, at the same time, the data based on estimations is another source for inconsistency. First, third-party estimation methods vary between and within each provider (e.g., Thomson Reuters applies one of three different estimation methods depending on data availability). This explains why the results of third-party estimations are rather inconsistent. Second, the underlying assumptions and estimation rules are not fully disclosed in a transparent manner. This explains why it remains challenging from an outsider's perspective to determine the reasons for the prevailing inconsistency in full detail. Notably, it remains not clear what data providers do and do not include in their Scope 3 estimations.

Most data providers use estimations based on input-output analyses. The advantage for third-party providers is that the data are publically available and user-friendly (Huang et al., 2009). However, the clear disadvantage is that results remains an estimate based on industry or sector averages, that is, you cannot detect the good performers from the laggards within any given sector (Minx et al., 2009). Process analysis could mitigate this issue; however, it requires a lot of company and process-specific information, and thus, it is likely to play rather a minor role for data providers who need to handle huge amounts of data points. In sum, third-party estimates make CCP data more comprehensive and wide-ranging, closing data gaps specifically regarding supply chains and small and medium-sized enterprises (Comas Martí \& Seifert, 2013). It appears very likely that stakeholders will have to rely on third-party estimated data in the coming years. In terms of data consistency, we derive two implications: First, data providers should use a combination of the most reliable estimation methods-according to data availability. Second, providers should disclose in more detail how the estimations are conducted.

\section{I CONCLUSION}

These results on the consistency of CCP data between third-party providers are relevant for future research, corporate management, financial markets, data providers as well as policymaking. First, academic researchers must be mindful of the consistency of CCP data for future research settings, as it can significantly affect the results. We would advise researchers to apply three practicable steps to increase the consistency of CCP data in future studies. First, extreme outliers should be examined to see if they are errors or actual outliers. For example, a company using renewable energy may have zero Scope 1 emissions - so the provided data are indeed an outlier but nevertheless correct and, thus, should be included. Second, scholars should repeat their analyses for data sets with and without outliers. The results show whether the outliers make a difference and to which scale they affect the results. The third step is to carefully select the source of CCP data, not just focusing on emission scopes, but also considering the differences of corporate-reported data and third-party estimation methods. If researchers are able to distinguish the two sources of data, we would suggest reporting the results in different models. For example, one model could cover for the entire sample and one model could cover only the company-reported data as a robustness check. Furthermore, future research could test the correlations of third-party estimated CCP data between the provider amounts and the maximum industry adjusted amount, also known as the Engaged Tracking amounts. The Engaged Tracking data (ET Carbon Dataset) has been used in several recent studies (Belkhir \& Elmeligi, 2019; Jackson \& Belkhir, 2018).

Second, corporate management should avoid situations where different CCP data are communicated or presented externally. Management should be very careful to check the consistencies in the CCP data, including the CDP and their own sustainability reports. The tolerated latitude of carbon accounting discourages consistent data are reflected in the inconsistencies between third-party data providers. Furthermore, companies should be aware of the release of CCP data based on third-party estimation methods, notably in the Scope 3 context. Managers could start 
gathering and disclosing Scope 3 emissions on their own as well as become more involved in improving standards for related estimation methods. They should join in a wider discourse on transparency and clarity of CCP data, especially what are the most consistent approaches for all scopes. Furthermore, with respect to Scope 3 emissions, it would be desirable to develop mutually agreed minimum standards. In sum, data consistency in Scope 3 would particularly benefit from a universally accepted standardization. Eventually, extending the scope of coverage to include small companies would also benefit from user-friendly accounting tools and reporting standards for these firms (Bradford \& Fraser, 2008).

Third, our results point toward a challenge for a key player in the future decarbonization process, financial markets. On the one hand, CCP data are becoming increasingly relevant for investment appraisals (Depoers et al., 2016; Matsumura et al., 2014; Reid \& Toffel, 2009). On the other hand, the consistency of data is not guaranteed, and could be hindering adequate analyses and assessments. Investors should engage firms to follow a standardized approach accordingly. Moreover, it is important to further raise the awareness in financial markets that capturing climate risks in investment portfolios must go beyond pure carbon footprints. Ex-post data about carbon performance on its own typically does not reveal any information for future risks. Further information about individual assets-such as their carbon dependency, decarbonizing options, and adaptation-related exposures-is required (Diaz-Rainey et al., 2017). Thus, carbon footprints are an essential step of a holistic climate risk management approach within investment appraisals.

Fourth, data providers should increase the transparency about their own estimation methods. We are aware that further standardization is not the most likely way forward regarding third-party estimation methods. It is well known that different estimation methods inherently yield different and, thus, inconsistent results. As our analysis shows, the correlation results for combined Scope 1 and 2 emissions for third-party estimated data do not diverge too greatly from reported data. As such, estimating CCP appears to be a promising way to close related data gaps. However, the data provider could help by increasing transparency of the details of their estimation methods. For example, data providers could indicate to which extend and in which cases they use sector-averages to infer firm-level emissions. Thus, investors can decide which third-party estimation methods they find most adequate and reliable. Eventually, the argument to move beyond publicly listed firms is also valid in the estimation context: phasing in estimations for firms other than large listed corporations will be an important step in order to pave the way for an economy-wide decarbonization.

Fifth, the findings of this article are also relevant for policymakers. For example, the recently released "Action Plan: Financing Sustainable Growth" of the EU Commission (2018a) seeks to reorient capital flows toward sustainable investment and manage financial risks stemming from climate change. It intends to develop sustainability benchmarks, which is only possible with a sound and standardized methodology to calculate carbon footprints. Furthermore, risks associated with climate change shall play an important role. All these ambitious efforts require high quality CCP data. This article illustrates some current shortcomings and severe challenges ahead.

\section{CONFLICT OF INTEREST}

The authors declare no conflict of interest.

\section{ORCID}

Timo Busch (iD https://orcid.org/0000-0001-6405-5252

Matthew Johnson (iD https://orcid.org/0000-0001-6491-2082

\section{REFERENCES}

Andrew, J., \& Cortese, C. L. (2011). Carbon disclosures: Comparability, the carbon disclosure project and the greenhouse gas protocol. Australasian Accounting, Business and Finance Journal, 5(4), 5-18.

Belkhir, L., \& Elmeligi, A. (2019). Carbon footprint of the global pharmaceutical industry and relative impact of its major players. Journal of Cleaner Production, 214, 185-194.

Block, C., vanPraet, B., Windels, T., Vermeulen, I., Dangreau, G., Overmeire, A., ... Vandecasteele, C. (2011). Toward a carbon dioxide neutral industrial park: A case study. Journal of Industrial Ecology, 15(4), 584-596.

Bloomberg Finance L.P. (2019). Bloomberg Database. Retrieved from Bloomberg Terminal. Accessed 20 Jun 2018.

Bowen, F., \& Wittneben, B. (2011). Carbon accounting: Negotiating accuracy, consistency and certainty across organisational fields. Accounting, Auditing \& Accountability Journal, 24(8), 1022-1036.

Bradford, J., \& Fraser, E. D. (2008). Local authorities, climate change and small and medium enterprises: Identifying effective policy instruments to reduce energy use and carbon emissions. Corporate Social Responsibility and Environmental Management, 15(3), 156-172.

Burkhardt, J. J., Heath, G., \& Cohen, E. (2012). Life cycle greenhouse gas emissions of trough and tower concentrating solar power electricity generation. Journal of Industrial Ecology, 16, 93-109.

Busch, T., \& Hoffmann, V. H. (2011). How hot is your bottom line? Linking carbon and financial performance. Business \& Society, 50(2), $233-265$.

Busch, T., \& Lewandowski, S. (2018). Corporate carbon and financial performance: A meta-analysis. Journal of Industrial Ecology, 22(4), 745-759.

Carbon Disclosure Project. (2017). CDP 2007-2017 Annual Questionnaire - Public Responses. Accessed 29 Jan 2018.

CDP. (2010). S\&P 100 Carbon Chasm: Analysis based on Carbon Disclosure Project 2007 - 2009 Responses Drawn from the USA's 100 Largest Companies. Retrieved from https://www.cdproject.net. Access ed 26 July 2018.

CDP. (2017). CDP Scores 2017. Retrieved from https://www.cdp.net/en/scores-2017. Accessed November 20, 2018. 
Clarkson, P. M., Li, Y., Richardson, G. D., \& Vasvari, F. P. (2008). Revisiting the relation between environmental performance and environmental disclosure: An empirical analysis. Accounting, Organizations and Society, 33, 303-327.

Comas Martí, M. J., \& Seifert, R. W. (2013). Assessing the comprehensiveness of supply chain environmental strategies. Business Strategy and the Environment, 22(5), 339-356.

ConocoPhilips (2011). Sustainable Development Report 2010. Retrieved from http://www.conocophillips.com/company-reports-resources/sustainabilityreporting/sustainability-report-regional-archives/. Accessed December 12, 2019.

Damert, M., Paul, A., \& Baumgartner, R. J. (2017). Exploring the determinants and long-term performance outcomes of corporate carbon strategies. Journal of Cleaner Production, 160, 123-138.

Depoers, F., Jeanjean, T., \& Jérôme, T. (2016). Voluntary disclosure of greenhouse gas emissions: Contrasting the carbon disclosure project and corporate reports. Journal of Business Ethics, 134(3), 445-461.

Diaz-Rainey, I., Robertson, B., \& Wilson, C. (2017). Stranded research? Leading finance journals are silent on climate change. Climatic Change, 143(1), 243260.

Dolan, S. L., \& Heath, G. A. (2012). Life Cycle Greenhouse Gas Emissions of Utility-Scale Wind Power. Journal of Industrial Ecology, $16($ S1), $136-154$.

Downie, J., \& Stubbs, W. (2013). Evaluation of Australian companies' Scope 3 greenhouse gas emissions assessments. Journal of Cleaner Production, 56, 156163.

Dragomir, V. D. (2012). The disclosure of industrial greenhouse gas emissions: A critical assessment of corporate sustainability reports. Journal of Cleaner Production, 29, 222-237.

Enbridge Inc. (2017). Sustainability Report 2016. Retrieved from https://www.enbridge.com/Sustainability-Reports/sustainability-report/ /media/CSR2/ pdf/2016_CSR_Report.pdf. Accessed Jan 20, 2020.

EPA. (2017). Greenhouse Gas Reporting Program (GHGRP). Key Facts and Figures. Retrieved from https://www.epa.gov/ghgreporting/key-facts-andfigures. Accessed June 24, 2019.

EPA. (2018). Greenhouse Gas Reporting Program and the U.S. Inventory of Greenhouse Gas Emissions and Sinks. Retrieved from https://www.epa.gov/ ghgreporting/greenhouse-gas-reporting-program-and-us-inventory-greenhouse-gas-emissions-and-sinks. Accessed Nov $28,2018$.

EU Commission. (2018a). Action Plan: Financing Sustainable Growth. Retrieved from https://eur-lex.europa.eu/legal-content/EN/TXT/?uri=CELEX: 52018DC009. Accessed July 15, 2018.

EU Commission. (2018b). EU Emission Trading System (EU ETS). Retrieved from https://ec.europa.eu/clima/policies/ets_en. Accessed Nov 20, 2018.

Goldhammer, B., Busse, C., \& Busch, T. (2017). Estimating corporate carbon footprints with externally available data. Journal of Industrial Ecology, 21(5), 11651179.

Gooding, C. H. (2012). Data for the carbon footprinting of rendering operations. Journal of Industrial Ecology, 16(2), $223-230$.

Griffin, P. A., Lont, D. H., \& Sun, E. Y. (2017). The relevance to investors of greenhouse gas emission disclosures. Contemporary Accounting Research, 34(2), 1265-1297.

Hahn, R., Reimsbach, D., \& Schiemann, F. (2015). Organizations, climate change, and transparency: Reviewing the literature on carbon disclosure. Organization \& Environment, 28(1), 80-102.

Huang, Y. A., Lenzen, M., Weber, C. L., Murray, J., \& Matthews, H. S. (2009). The role of input-output analysis for the screening of corporate carbon footprints. Economic Systems Research, 21(3), 217-242.

Hummel, K., \& Schlick, C. (2016). The relationship between sustainability performance and sustainability disclosure-Reconciling voluntary disclosure theory and legitimacy theory. Journal of Accounting and Public Policy, 35(5), 455-476.

ISS-Ethix Climate Solutions. (2019). ISS ESG Database. Accessed 2 Aug 2018.

Jackson, J., \& Belkhir, L. (2018). Assigning firm-level GHGE reductions based on national goals-Mathematical model \& empirical evidence. Journal of Cleaner Production, 170, 76-84.

Kolk, A., Levy, D., \& Pinkse, J. (2008). Corporate responses in an emerging climate regime: The institutionalization and commensuration of carbon disclosure. European Accounting Review, 17(4), 719-745.

Larsen, H. N., Solli, C., \& Pettersena, J. (2012). Supply chain management-How can we reduce our energy/climate footprint?Energy Procedia, $20,354-363$.

Lee, K.-H., \& Cheong, I.-M. (2011). Measuring a carbon footprint and environmental practice: The case of Hyundai Motors Co. Industrial Management \& Data Systems, 111(6), 961-978.

Lundie, S., Wiedmann, T., Welzel, M., \& Busch, T. (2019). Global supply chains hotspots of a wind energy company. Journal of Cleaner Production, 210, 10421050.

Luo, L., Lan, Y. C., \& Tang, Q. (2012). Corporate incentives to disclose carbon information: Evidence from the CDP Global 500 report. Journal of International Financial Management \& Accounting, 23(2), 93-120.

Marland, G., Buchholz, T., \& Kowalczyk, T. (2013). Accounting for carbon dioxide emissions: The context and stakeholders matter. Journal of Industrial Ecology, 17(3), 340-342.

Matisoff, D. C., Noonan, D. S., \& O’Brien, J. J. (2013). Convergence in environmental reporting: Assessing the Carbon Disclosure Project. Business Strategy and the Environment, 22(5), 285-305.

Matsumura, E. M., Prakash, R., \& Vera-Munoz, S. C. (2014). Firm-value effects of carbon emissions and carbon disclosures. The Accounting Review, 89(2), 695724.

Mazor, M. H., Mutton, J. D., Am Russell, D., \& Keoleian, G. A. (2011). Life cycle greenhouse gas emissions reduction from rigid thermal insulation use in buildings. Journal of Industrial Ecology, 15(2), 284-299.

Minx, J. C., Wiedmann, T., Wood, R., Peters, G. P., Lenzen, M., Owen, A., ... Baiocchi, G. (2009). Input-output analysis and carbon footprinting: An overview of applications. Economic Systems Research, 21(3), 187-216.

MSCI ESG Research. (2019). MSCI ESG CarbonMetrics. Accessed May 8, 2018.

Perrault, E., \& Clark, C. (2010). Should corporate social reporting be voluntary or mandatory? Evidence from the banking sector in France and the United States. Corporate Governance: The International Journal of Business in Society, 10(4), 512-526.

Reid, E. M., \& Toffel, M. W. (2009). Responding to public and private politics: Corporate disclosure of climate change strategies. Strategic Management Journal, 30(11), 1157-1178. 
Rekker, S. A., Humphrey, J. E., \& O’Brien, K. R. (2019). Do sustainability rating schemes capture climate goals?Business \& Society, https://doi.org/10.1177/ 0007650319825764.

Schaltegger, S., \& Csutora, M. (2012). Carbon accounting for sustainability and management. Status quo and challenges. Journal of Cleaner Production, 36 , 1-16.

Stanny, E., \& Ely, K. (2008). Corporate environmental disclosures about the effects of climate change. Corporate Social Responsibility and Environmental Management, 15, 338-348.

Stanny, E. (2018). Reliability and Comparability of GHG Disclosures to the CDP by US Electric Utilities. Social and Environmental Accountability Journal, 1-20. Suh, S., Huppes, G., \& (2009). Methods in the life cycle inventory of a product. In S.Suh (Ed.), Handbook of input-output economics in industrial ecology. Springer. Sullivan, R., \& Gouldson, A. (2012). Does voluntary carbon reporting meet investors' needs? Journal of Cleaner Production, 36, 60-67.

Sustainalytics. (2019). Sustainalytics Data Services. Accessed Jun 122018.

Talbot, D., \& Boiral, O. (2018). GHG reporting and impression management: An assessment of sustainability reports from the energy sector. Journal of Business Ethics, 147(2), 367-383.

TCFD. (2017). Recommendations of the Task Force on climate-related financial disclosures. Retrieved from https://www.fsb-tcfd.org/publications/finalrecommendations-report/. Accessed February 20, 2019.

Thomson Reuters ESG. (2019). Thomson Reuters Database. Retrieved from Thomson Reuters EIKON. Accessed Apr 112018.

Trucost ESG Analysis. (2019). Trucost Database. Accessed 2 Mar 2018.

WCMD. (2019). World Carbon Market Database. The carbon disclosure solution. Retrieved from https://www.carbonmarketdata.com/en/home. Accessed January 15, 2019.

Wiedmann, T. (2009). A review of recent multi-region input-output models used for consumption-based emission and resource accounting. Ecological Economics, 69(2), 211-222.

Wiedmann, T., \& Minx, J. (2008). A definition of 'carbon footprint'. Ecological Economics Research Trends, 1, 1-11.

WRI/WBCSD. (2004). The Greenhouse Gas Protocol. A corporate accounting and reporting standard (revised edition). Geneva, Switzerland: WRI/WBCSD. (ISBN 978-1-56973-772-9).

\section{SUPPORTING INFORMATION}

Additional supporting information may be found online in the Supporting Information section at the end of the article.

How to cite this article: Busch T, JohnsonM, PiochT. Corporate carbon performance data: Quo vadis? J Ind Ecol. 2020;1-14. https://doi.org/10.1111/jiec.13008 